\title{
A SAW TOOTH INSTABILITY MODEL IN STORAGE RINGS
}

\author{
M. Migliorati ${ }^{*}$ L. Palumbo, University of Rome - Dip. Energetica, Italy \\ G. Dattoli, L. Mezi, ENEA, Dip. Innovazione, Frascati, Rome, Italy
}

\begin{abstract}
The time evolution of the energy spread above the microwave instability threshold in a storage ring is described by means of two coupled non linear differential equations. The results are in agreement with a time domain simulation code. The model equations are combined to those accounting for the storage ring Free Electron Laser dynamics, in order to study the interplay between the FEL and the storage ring instability dynamics.
\end{abstract}

\section{INTRODUCTION}

The microwave instability produces an increase of energy spread and a consequent anomalous bunch lengthening, and limits the performances of storage rings. It has been the subject of several investigations [1, 2, 3, 4], and the problem has been studied by exploiting the linearized Vlasov equation, which describes the time evolution of the single bunch distribution function in the phase space. The threshold of the instability depends on the wake fields and on the current intensity. Above the threshold the linear theory can not explain the time evolution of the distribution function. The increase of the bunch phase space dimensions may provide the conditions for a new equilibrium configuration. It may happens, however, that the equilibrium is never reached, and the energy spread oscillates in time with a pattern similar to relaxation type oscillations, as shown in Fig. 1. Such a behavior is known as saw tooth instability [4], and it occurs in storage rings characterized by intense beam currents.

An appropriate analysis of the saw tooth instability, requires the inclusion of non linear terms in the Vlasov equation. The problem has been afforded in ref. [5], where it is shown that the time evolution of the distribution function may have different patterns, including relaxation type oscillations.

In this paper, analyzing the results of appropriate numerical simulations, we study the conditions for the onset, growth and relaxation of the saw tooth oscillations for a pure inductive impedance. The analysis points out the mechanisms responsible for the saw tooth behavior: the growth of the instability is produced by non linear wakes, it is counteracted by the Landau damping, and it is finally damped by the radiation process. We describe these effects through some quantities averaged over the bunch distribution, giving a model employing two coupled non linear equations which reproduce the relaxation oscillations of the instability in a fairly satisfactory way. We employ numerical simulations to show that a Free Electron Laser (FEL),

\footnotetext{
*Email: migliorati@axrma.uniroma1.it
}

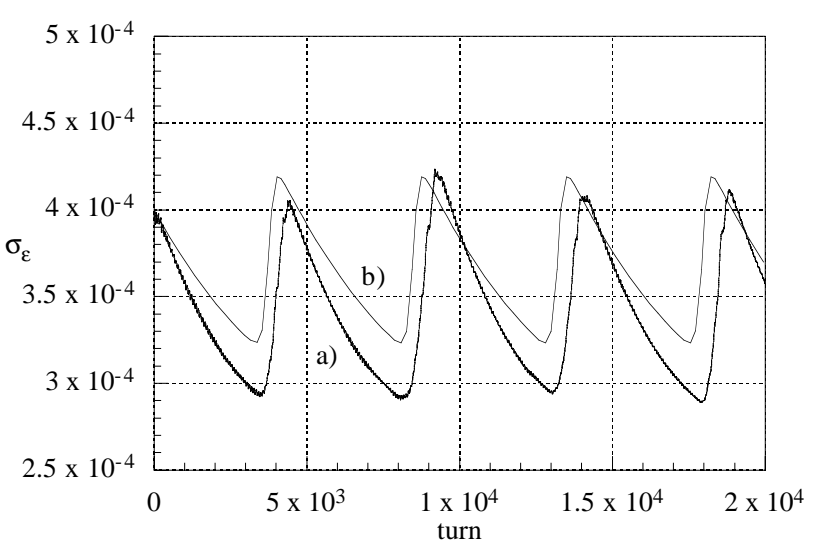

Figure 1: Energy spread vs number of turns: a) simulations, b) model equations.

used either as an amplifier or an oscillator, may be an efficient tool to damp the saw tooth instability. The physical interpretation of the simulation results has also suggested the way to extend the model equations by including the effects of the FEL in the oscillator mode. This approach provides a clear picture of the FEL-storage ring mutual feedback, and clarifies the mechanism of the instability inhibition.

\section{SAW TOOTH INSTABILITY}

For the study of the saw tooth instability, we use a time domain simulation code, which includes the effects of the self induced wake fields. The simulations, using a pure inductive impedance and employing $3 \times 10^{5}$ macroparticles, show a clear saw tooth behavior (Fig. 1, a) line) above the microwave threshold. The evolution of the phase space distribution gives further insight for a better understanding of the mechanisms responsible for the growth of the energy spread and of its damping. In fact, referring to Fig. 1, when the energy spread is close to one minimum, the phase space distribution shows a perturbation, superimposed to the stationary distribution, which suggests the existence of a coherent microstructure (microbunching) inside the bunch [6]. This microbunching increases the energy spread producing a turbulent distribution. In some regions the particles, due to the wake fields non linearities, experience larger oscillation amplitudes, and the distribution endures a filamentation process. The phase space evolution is then characterized by a reduction of the local density due to the particle diffusion which tends to decrease the microbunching efficiency so that, the natural radiation and Landau dampings are able to counteract the 
phase space anomalous growth, until the initial configuration is restored.

The microbunching mechanism producing the microwave instability has been discussed in ref. [7], where it is considered the responsible for coherent radiation emission observed in operating storage rings affected by strong microwave instability, an effect similar to the Free Electron Laser dynamics.

The analysis of the simulations clarifies also the contribution of the damping mechanisms: the radiation process is the main responsible for the saw tooth behavior, while the Landau damping is important in counteracting the instability. In fact, by running simulations with a very long radiation damping time, the saw tooth pattern disappears, the bunch distribution remaining stable with an energy spread slightly larger than the maximum obtained from Fig. 1.

\section{DYNAMICAL MODEL}

To model the saw tooth behavior, we start by considering the coasting beam case, for which the linear theory predicts an instability caused by a growth of a perturbation produced by the wake fields, and an opposed damping deriving from a spread in the oscillation frequencies (Landau damping) [1]. The extension of the theory to the bunched beam case is known as Boussard criterion [8].

If we suppose that the energy spread $\sigma_{\varepsilon}$ is a quadratic combination of the natural $\left(\sigma_{\varepsilon n}\right)$ and instability induced $\left(\sigma_{i}\right)$ parts, that is

$$
\sigma_{\varepsilon}=\left(\sigma_{\varepsilon n}^{2}+\sigma_{i}^{2}\right)^{\frac{1}{2}}=\sigma_{\varepsilon n}\left(1+\sigma_{r}^{2}\right)^{\frac{1}{2}}
$$

and consider a Gaussian bunch distribution with a Lorentz spectrum of the oscillation frequencies, then the growth rate of the instability induced by the wake fields can be written as [6]

$$
\frac{n}{T_{0}} \sqrt{\frac{(2 \pi)^{3 / 2} I_{0} \nu_{s}|Z / n|}{\left(E_{0} / e\right) \sigma_{\varepsilon n}}} \frac{1}{\left(1+\sigma_{r}^{2}\right)^{\frac{1}{4}}}=\frac{A}{\left(1+\sigma_{r}^{2}\right)^{\frac{1}{4}}}
$$

and the Landau term as

$$
\frac{n}{T_{0}} 2 \pi \alpha_{c} \sigma_{\varepsilon n}\left(1+\sigma_{r}^{2}\right)^{\frac{1}{2}}=B\left(1+\sigma_{r}^{2}\right)^{\frac{1}{2}}
$$

where $n$ is an harmonic of the revolution frequency, $\alpha_{c}$ the momentum compaction, $T_{0}$ the revolution period, $I_{0}$ the average beam current, $Z / n$ the broad band impedance at the $n^{t h}$ harmonic of the revolution frequency, $E_{0}$ the beam energy.

We derive now differential equations, governing the time evolution of the energy spread and of the saw tooth growth rate, without entering into the details of the phase space dynamics. We denote the growth rate of the instability by $\alpha$ and relate such a quantity with the induced energy spread $\sigma_{r}$, according to the obvious relation

$$
\frac{1}{\sigma_{r}^{2}} \frac{d \sigma_{r}^{2}}{d t}=\left(\alpha-\frac{2}{\tau_{s}}\right)
$$

where $\tau_{s}$ is the longitudinal damping time.

To explain the saw tooth behavior, we assume that $\alpha$ is a time dependent function. Physical observations suggest that the equation should include the Boussard criterion stationary solution, thus yielding a dependence of the energy spread on the current as $I_{0}^{1 / 3}$. The equation should also be capable of reproducing relaxation type oscillations. From these considerations, we make the ansatz that $\alpha$ satisfies an equation of the type

$$
\frac{1}{\alpha} \frac{d \alpha}{d t}=\frac{c_{1} A}{\left(1+\sigma_{r}^{2}\right)^{\frac{1}{4}}}-c_{2} B\left(1+\sigma_{r}^{2}\right)^{\frac{1}{2}}
$$

which contains, on the right hand side, the two opposite terms (2) and (3), accounting respectively for the wake fields instability and the Landau damping, $c_{1}$ and $c_{2}$ being constant parameters.

Eqs. (4) and (5) provide our model equations. They give stationary solutions with $\sigma_{\varepsilon}=\sigma_{\varepsilon n}$ in any situation for which $c_{1} A<c_{2} B$. The case $c_{1} A=c_{2} B$ is exactly the Boussard criterion which is therefore satisfied.

The above equations rely on simple assumptions. For example, in case of the $A$ parameter, the actual bunch shape is distorted by the potential well and is not Gaussian. Furthermore a local density perturbation may be responsible for the microwave instability [7]. This is the reason we introduced the factor $c_{1}$ in eq. (5).

For what concern the $B$ parameter, and then the introduction of the factor $c_{2}$, the major approximations are in the linear relationship assumed between the spectrum width producing Landau damping and the energy spread $\sigma_{\varepsilon}$, and the assumption of a Lorentz spectrum. Actually the oscillation frequency spectrum depends on the bunch distribution and on the non linearities of the wake fields.

To verify the model, we first estimate the constants $c_{1}$ and $c_{2}$ by comparing the simulations and the solution of Eqs. (4) and (5) under the condition of stability, that is for $c_{1} A<c_{2} B$, getting, as best fit, $c_{1}=0.35$ and $c_{2}=2.1$. Then, with the same factors $c_{1}$ and $c_{2}$, we analyze the case of instability. The resulting time behavior of the energy spread, given by Eqs. (4) and (5), is shown in Fig. 1 (the b) line), which shows a good agreement with the simulation results. If we increase the damping time $\tau_{s}$ and eventually it tends to infinity, the saw tooth behavior disappears, thus giving a stationary energy spread which depends on the initial conditions and on the coefficients $c_{1} A$ and $c_{2} B$, that is on the intensity of both the instability and Landau damping. In this case, from eq. (5), we get that the energy spread has a dependence on the current of the kind $I_{0}^{1 / 3}$. 


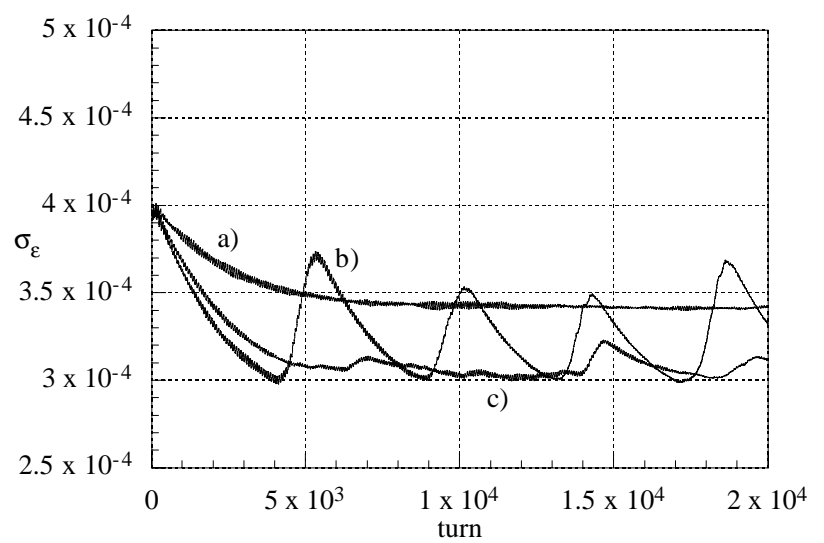

Figure 2: Energy spread vs number of turns with the FEL amplifier for different FEL intensities.

\section{FEL CONTRIBUTION}

We have investigated the effect of the FEL on the storage ring saw tooth instability. The FEL induced energy spread and associated bunch lengthening decrease the peak current and the wake field intensity, thus shifting the instability threshold to higher levels. The parameter which can be used to control the instability is the intensity of the laser field itself. In Fig. 2 we have considered a storage ring FEL amplifier. When the laser intensity is sufficiently large (the a) curve), the FEL interaction destroys completely the saw tooth instability pattern, the beam energy spread is essentially that induced by the FEL. For lower intensity the saw tooth oscillation amplitudes are reduced (the b) curve), for intermediate intensity (the c) curve) it is possible to switch off the instability without inducing any energy spread.

A similar analysis has been performed with the FEL in the oscillator mode. In this case, the extension of the model to include the FEL dynamics can be useful to provide an explanation of the complex phenomenology of the interplay between the FEL and the storage ring by means of simple but physically pregnant and transparent formulae. By recalling that the FEL induced energy spread, proportional to the laser power, combines quadratically to the natural one [9], eq. (4) is modified in

$$
\frac{d \sigma_{r}^{2}}{d t}=\alpha \sigma_{r}^{2}-\frac{2}{\tau_{s}}\left(\sigma_{r}^{2}-x_{0}\right)
$$

where $x_{0}$ is linked to the intracavity power and satisfies the differential equation

$$
\begin{aligned}
& \frac{1}{x_{0}} \frac{d x_{0}}{d t}=\frac{.85 g_{0}}{T_{0}} \\
& \quad\left[\frac{1}{\sqrt{1+\sigma_{r}^{2}}+1.7 \mu_{\varepsilon}(0)^{2}\left(1+\sigma_{r}^{2}\right)^{3 / 2}}-\frac{\eta}{.85 g_{0}}\right]
\end{aligned}
$$

where $g_{0}$ is the FEL small signal gain coefficient, $\mu_{\varepsilon}(0)=$ $4 N \sigma_{\varepsilon n}$ with $N$ being the number of ondulator periods,

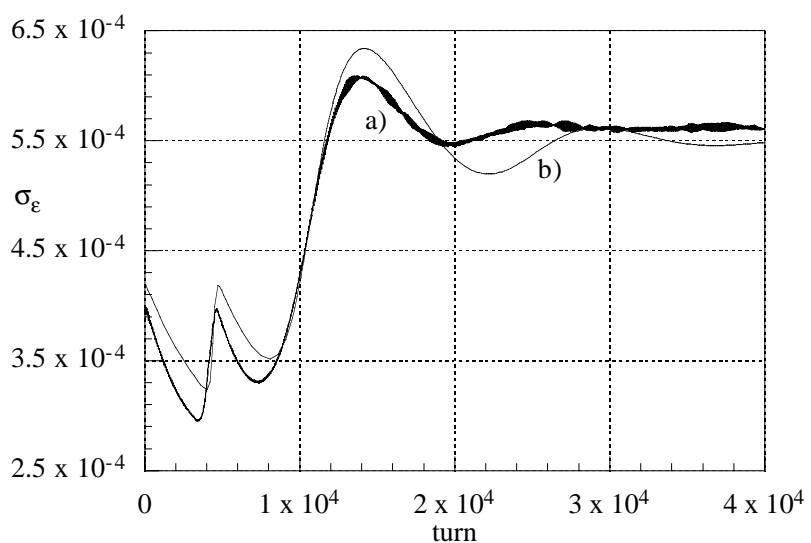

Figure 3: Energy spread vs number of turns with the FEL oscillator.

and $\eta$ represents the cavity losses. The results of the coupled equations in presence of the FEL oscillator show that the saw tooth instability is critical to the ratio between the losses and the gain. If this ratio is relatively small, the intracavity power grows and induces a large energy spread which overcomes the saw tooth instability. Then the growth rate $\alpha$ is suddenly brought to zero and the total energy spread tends to a stable behavior. When the ratio increases, the onset of the laser takes longer time to establish. In Fig. 3 we show a preliminary comparison between the results of the model (the b) line) and the more time consuming numerical simulations (the a) line). The agreement is fairly satisfactory, and further investigations are in progress.

\section{REFERENCES}

[1] A. W. Chao, Physics of Collective Beam Instabilities in High Energy Accelerators, Wiley-Interscience, New York (1993).

[2] K. Oide, and K. Yokoya, KEK Report 90-10, KEK, Tsukuba, Japan (1990).

[3] K. L. F. Bane, and K. Oide, in Proc. IEEE Particle Accelerator Conference, Washington, (1994).

[4] M. D'yachkov, Ph.D. thesis, University of British Columbia, (1995).

[5] G. V. Stupakov, et al., Physical Review E 55, 5976 (1997).

[6] G. Dattoli, et al., inProc. of 16th ICFA Workshop on Nonlinear and Collective Phenomena in Beam Physics, Arcidosso, Italy, (1998).

[7] J. M. Wang, Physical Review E 58, 984 (1998).

[8] D. Boussard, Technical Report No. LABII/RF/Int./75-2, CERN, Geneva, (1975).

[9] G. Dattoli, et al., NIM A 393, (1997). 